\title{
Longitudinal current in single-atom interacting with laser field
}

\author{
Anatoli Andreev ${ }^{1}$, Sergei Stremoukhov ${ }^{1,2, *}$, and Olga Shoutova ${ }^{1}$ \\ ${ }^{1}$ Faculty of Physics, Lomonosov Moscow State University, 119991, Moscow, Russia \\ ${ }^{2}$ National Research Centre "Kurchatov Institute”, 123182, Moscow, Russia
}

The progress of the scientific research in the last century shows the following: the macroscopic phenomena and devices based on them find soon their realizations in microphysics. That is the case of the one-atom lasing device, the one-electron transistor, etc. Here, we discuss another phenomenon of such kind, the longitudinal current in a single atom [1,2]. Such generalization of the longitudinal wave concept is absolutely self -consistent. The atomic current density is strongly defined in material wave theory. By calculating the atomic current density we can divide it into the transversal and longitudinal parts which are orthogonal and parallel to the driving laser field wave vector, respectively. The appearance of the longitudinal waves or currents is usually associated with structured fields, i.e. tightly focused beams, evanescent or interference fields. In the case of single atom the appearance of the longitudinal component in atomic current is due to the breaking of spherical symmetry when the atomic electron interacts with superposition of intra-atomic field and the field of external wave. Indeed the superposition of intra-atomic field and field of the external plane wave ceases to be the spherically symmetric. The non-perturbative theory of light-atom interaction [3] enables us to calculate the response field polarization and what is more important the dependency of polarization of emitted waves on the polarization of the driving laser field.

The effect of the high order harmonic generation $(\mathrm{HHG})$ in the intense laser fields is under permanent study for the three last decades. During this long period a number of different theoretical models have been proposed (see for review [3]). Most commonly the three-step model is used. This model is more semi-intuitive than self-consistent, as it combines the quantum process of ionization, the classical evolution of ionized electron in the field of the laser pulse, and finally the recombination of accelerated electron on the parent ion. The common feature of these models consists in a supposition that the HHG is a process of inelastic laser field scattering by atom, molecule, ion, etc. In analogy with the early models of the second or third harmonic generation it is assumed that the energy of a harmonic is a sum of energies of laser radiation quanta. For example, the three quantum process $\omega_{3}=n_{1} \omega_{1}+n_{2} \omega_{2}$ in femtosecond two-colour field $\omega_{1}=\omega$ and $\omega_{2}=2 \omega$ results in fourth harmonic generation at $n_{1}=2, n_{2}=1$ or in terahertz radiation generation at $n_{1}=2, n_{2}=-1$, etc.

The alternative approach to the theory of light-atom interaction was proposed in [3]. This method is based on the use the new complete orthonormal basis for expansion of the time-dependent Schrodinger equation wave-function. The proposed basis consists of time-dependent eigenfunctions of boundary value problem for "an atom in the external field" [3]. Here, the HHG can be interpreted as a parametric process, and the energy of emitted harmonics results from the work produced by laser field on electron movement in the intra-atomic field. In the frames of this theory, the HHG in the double-frequency field is a process when the generation of $\mathrm{N}$-th harmonic is due to the interaction of the following type $N \omega=n_{1} \omega+n_{2} \cdot 2 \omega$, where $n_{1,2}$ can be both positive and negative.

In the frames of dipole approximation, it is assumed that the linearly polarized light interacts with $\mathrm{z}$ component of dipole moment while circularly polarized light interacts with $(x \pm i y)$ components

\footnotetext{
* Corresponding author: sustrem@gmail.com
} 
of atomic electron radius vector [5]. It should be noted that the orientation of the atomic configuration space relates to the angular momentum orientation: the quantum-mechanical average of the angular momentum $\langle n \ln |i| n l m\rangle=\dot{n}_{z} \hbar m$ is oriented along the $\mathrm{z}$ axis of atomic configurational space.

Here, we present the specific feature of the single atom response which can be consistently interpreted in the frames of the theory [3]: the appearance of the atomic current longitudinal component which is orthogonal to the polarization vector of the incident laser field. The longitudinal component appears even in the case of a single-color laser field. The effect opens a way towards the development of new methods of the generated radiation polarization control.

The origin of the longitudinal atomic current is in following. Indeed, the spatial distribution of the atomic electron density $\rho(\dot{r}, t)=e|\psi(\dot{r}, t)|^{2}$ is related to the atomic current density through the continuity equation $\partial \rho(\dot{r}, t) / \partial t+\operatorname{div} \dot{j}(\dot{r}, t)=0$. In the free atom case, when the orbital quantum number of ground state $l \geq 1$, the electron density distribution is cylindrically symmetric with respect to $\mathrm{z}$ axis of atomic configuration space. The direction of this axis coincides with the direction of the quantum-mechanical average $<i>$ of the atomic angular momentum. In the external laser field, the distribution of the electron density is influenced by the polarization of the driving laser field. Let us consider a spherically symmetric distribution of the electron density in s state. In the external laser field the electron density distribution takes the cylindrical symmetric form with an axis directed along the laser field polarization vector. The elongation of the electron density distribution in one direction obviously results in a contraction of the distribution radius in the perpendicular plane. This effect leads directly to the appearance of the longitudinal current or, more precisely, of the components of the atomic current perpendicular to the direction of the laser field polarization vector. It is evident that in the case of $l=0$ the integral longitudinal current is equal to zero due to the cylindrical symmetry of the electron density distribution in the external field. However, if $l>0$ and the angular momentum and field strength are not collinear, then the cylindrical symmetry is broken.

The results demonstrate clearly that the longitudinal component of the atomic current is efficiently induced when an atom with a non-zero angular momentum interacts with plane or one multicolour electromagnetic wave. This component is directed along the wave vector of the incident field and its amplitude depends on the geometry of the interaction, i.e. on the angles between the atomic angular momentum and the polarization vector (and wave vector) of the incident wave.

The spectrum of the longitudinal atomic current includes both odd and even harmonics of the incident field. The ratio of the amplitudes of odd and even harmonics depends on the orientation of the atomic angular momentum with respect to the polarization vector and wave vector of the driving laser field. So, we can easily control the spectrum of the HHG by varying the mutual orientation of the laser field polarization vector and the atomic angular momentum.

The account of the longitudinal component opens ways to control the spectral structure of the atomic current. It might be critical for the polarization state of the generated radiation. Usually, the detector system lies in line with the laser propagation direction [6], and the longitudinal projection of the current affect the final ellipticity only as an additional "dimension" which take energy away from the other projections. As a result, one can control the energy swop for the given harmonics. However, the impact of the longitudinal component can be more significant if the detector system has a non-zero angle with the laser field propagation direction. In that case one should take into account the longitudinal projection in ellipticity calculations. Account of the longitudinal projection can be critical for a consistent explanation of the results obtained in non-collinear geometries [7]. The work was partially supported by the RFBR under projects Nos. 18-02-00528, 18-02-00743, 18-02-40014.

\section{References}

1. A. Andreev, S. Stremoukhov, O. Shoutova, EPL, 120, 14003 (2017)

2. A. Andreev, S. Stremoukhov, O. Shoutova, JETP, 127, 25 (2018)

3. A. Andreev, S. Stremoukhov, O. Shoutova, Eur. Phys. J. D, 66, 16 (2012)

4. P. Corkum, Phys. Rev. Lett., 171, 1994 (1993)

5. L.D. Landau, E.M. Lifshitz, Quantum Mechanics (Pergamon Press, London-Paris, 1958)

6. S. Stremoukhov, A. Andreev, B. Vodungbo et al., Phys. Rev. A, 94, 013855 (2016)

7. M. Louisy, C. L. Arnold, M. Miranda et al., Optica, 2, 563, (2015) 